Recepción: 20 / 04 / 2017

Aceptación: 20 / 05 / 2017

Publicación: 15 / 07 / 2017

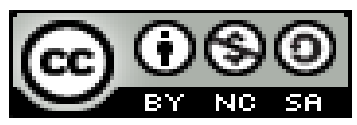

Ciencias Médicas

Reporte de Caso

\title{
Conveniencia de las hernioplastias de la pared abdominal con anestesia local
}

\author{
Convenience of abdominal wall hernioplasty with local anesthesia
}

\section{Correção de hérnias conveniência da parede abdominal sob anestesia local}

Rafael D. Espinosa-Cucalon ${ }^{\text {I }}$
danilo.espinosac@ug.edu.ec

Clara O. Jaime-Game II $^{2}$ clara.jaimeg@ug.edu.ec

Antonio Romero-Vargas ${ }^{\text {III }}$ antonio.romerov@ug.edu.ec

Carmen L. Marín-Soria IV carmen.marins@ug.edu.ec

Correspondencia: danilo.espinosac@ug.edu.ec

I. Diplomado en Docencia Superior; Especialista en Cirugía General; Diploma Superior en Diseño Curricular por Competencias; Magister en Docencia Universitaria e Investigación Educativa; Diplomado en Docencia Superior; Doctor en Medicina y Cirugía; Universidad de Guayaquil, Guayaquil, Ecuador.

II. Diplomado en Docencia Superior; Maestría en Docencia Universitaria e Investigación Educativa; Diploma Superior en Evaluación y Acreditación de la Educación Superior; Doctora en Medicina y Cirugía; Universidad de Guayaquil, Guayaquil, Ecuador.

III. Especialista en Ginecología y Obstetricia; Doctor en Medicina y Cirugía; Universidad de Guayaquil, Guayaquil, Ecuador.

IV. Diplomado en Docencia Superior; Magister en Educación Superior; Especialista en Gestión de Procesos Educativos; Obstetriz; Universidad de Guayaquil, Guayaquil, Ecuador. 


\section{Resumen}

Etimológicamente hernia proviene del latín equivalente a ruptura o del griego que implica yema o vástago.

Hernia es la protrusión de un órgano a través de la debilidad de la pared abdominal. Las hernias pueden ser asintomáticas y sintomáticas.

En las sintomáticas se presentan con mayor frecuencia: dolor, malestar relacionado con el contenido del saco herniario y alivio con el descanso.

En el examen físico es fundamental: Inspección y Palpación.

En la INSPECCIÓN, se puede observar con frecuencia la protrusión a manera de tumoración.

En la PALPACIÓN de la pared abdominal, se debe tocar el área implícita del abdomen o el orificio inguinal superficial, si es necesario para su identificación en esta región, utilizando la maniobra de Valsalva.

Palabras claves: Anestesia local; pared abdominal; hernioplastias. 


\section{Abstract}

Etymologically hernia comes from the Latin equivalent of rupture or from the Greek that implies bud or stem.

Hernia is the protrusion of an organ through weakness of the abdominal wall. Hernias can be asymptomatic and symptomatic.

In the symptomatic ones they present more frequently: pain, discomfort related to the contents of the hernial sac and relief with rest.

The physical examination is essential: Inspection and Palpation.

In the INSPECTION, the protrusion can be observed frequently as a tumor.

In the palpation of the abdominal wall, the implicit area of the abdomen or the superficial inguinal orifice should be touched, if necessary for identification in this region, using the Valsalva maneuver. Key words: Local anesthesia; abdominal wall; hernioplasties. 


\section{Resumo}

Etimologicamente, a hérnia vem do equivalente latino da ruptura ou do grego que implica broto ou caule.

A hérnia é a protrusão de um órgão através da fraqueza da parede abdominal. As hérências podem ser assintomáticas e sintomáticas.

Nos sintomas que apresentam mais freqüentemente: dor, desconforto relacionado ao conteúdo do saco hernial e alívio com descanso.

O exame físico é essencial: inspeção e palpação.

Na INSPECÇÃO, a protrusão pode ser observada frequentemente como um tumor.

Na palpação da parede abdominal, a área implícita do abdômen ou do orifício inguinal superficial deve ser tocada, se necessário para identificação nesta região, usando a manobra de Valsalva.

Palavras-chave: A anestesia local; parede abdominal; correção de hérnias. 


\section{Introducción.}

Identificado el defecto herniario, se deben solicitar exámenes elementales de laboratorio como: Hemograma, Glicemia, Urea, Creatinina, Tiempo de Protrombina, Tiempo de Tromboplastina Parcial, Micro Elisa, Hepatitis, Urianálisis y Valoración Cardiológica para preparar al paciente para el acto quirúrgico ${ }^{1-5}$.

La frecuencia con la que se presentan los distintos tipos de hernias es: Inguinales $75 \%$, Incisionales 10\%, Femorales 6\%, Umbilicales 3\%, Epigástricas 3\% y Otras $3 \%$ 5-6.

En el ámbito médico, siempre se ha minimizado la cirugía de hernias y es más, han sido asignados estos procedimientos, a quienes dan sus primeros pininos, garrafal error, que trae como consecuencia, recidivas que lamentar. Conviene puntualizar que, quién debe efectuar estas cirugías, son quienes tienen más conocimiento anatómico del área y del manejo de los tejidos ${ }^{7-9}$.

Las condiciones para que la reparación de la hernia se pueda efectuar con anestesia local son las siguientes $^{10-15}$.

1. Que el paciente, luego de las explicaciones pertinentes, prefiera este método.

2. Que el peso del paciente relacionado con la infiltración máxima permisible del anestésico den lugar a un procedimiento seguro.

3. Debe realizarse en quirófano.

4. Debe contar con el apoyo y la vigilancia del anestesiólogo.

5. Debe instalarse una vía venosa en el paciente y monitorización. 
6. Debe existir equipo de reanimación cardiopulmonar-cerebral disponible

El hecho de que el procedimiento se realice con anestesia local, no implica que no se guarden las seguridades necesarias para nuestro paciente.

La técnica anestésica para la región inguinal consiste en ${ }^{9-17}$ :

1. Bloqueo de los nervios iliohipogástrico e ilioinguinal, que son ramas de las raíces nerviosas T12-L1 e inervan el abdomen inferior, para lo cual, la infiltración del anestésico se la efectúa alejándose $2 \mathrm{cms}$. a la altura de la espina iliaca antero superior.

2. Infiltración en abanico, relacionada con la incisión a realizar.

3. Tiempo de espera corto de aproximadamente 5 minutos, que debe ir acompañado de la comprobación de la eficacia anestésica.

4. Guardar mínima cantidad de anestésico para infiltración en el cuello del saco herniario y alguna otra área que no esté adecuadamente anestesiada.

5. Siempre debe efectuarse aspiración frecuente para evitar inyectar anestésico directamente en un vaso sanguíneo. (Ver Fig. 1 y 2). 


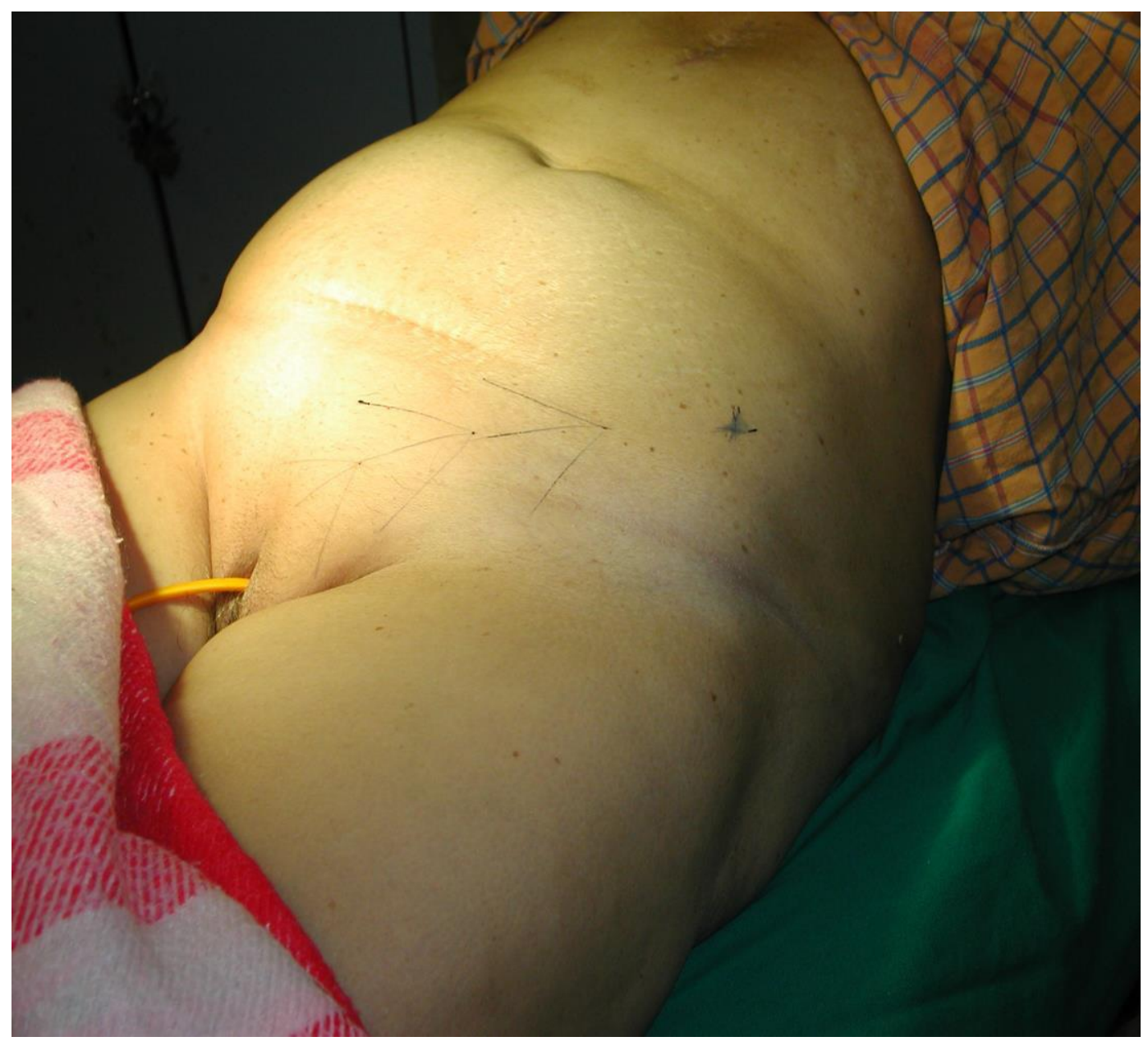

Figura $N^{\circ}$ 1.- Forma de realizar infiltración en abanico y punto de referencia del Bloqueo. 


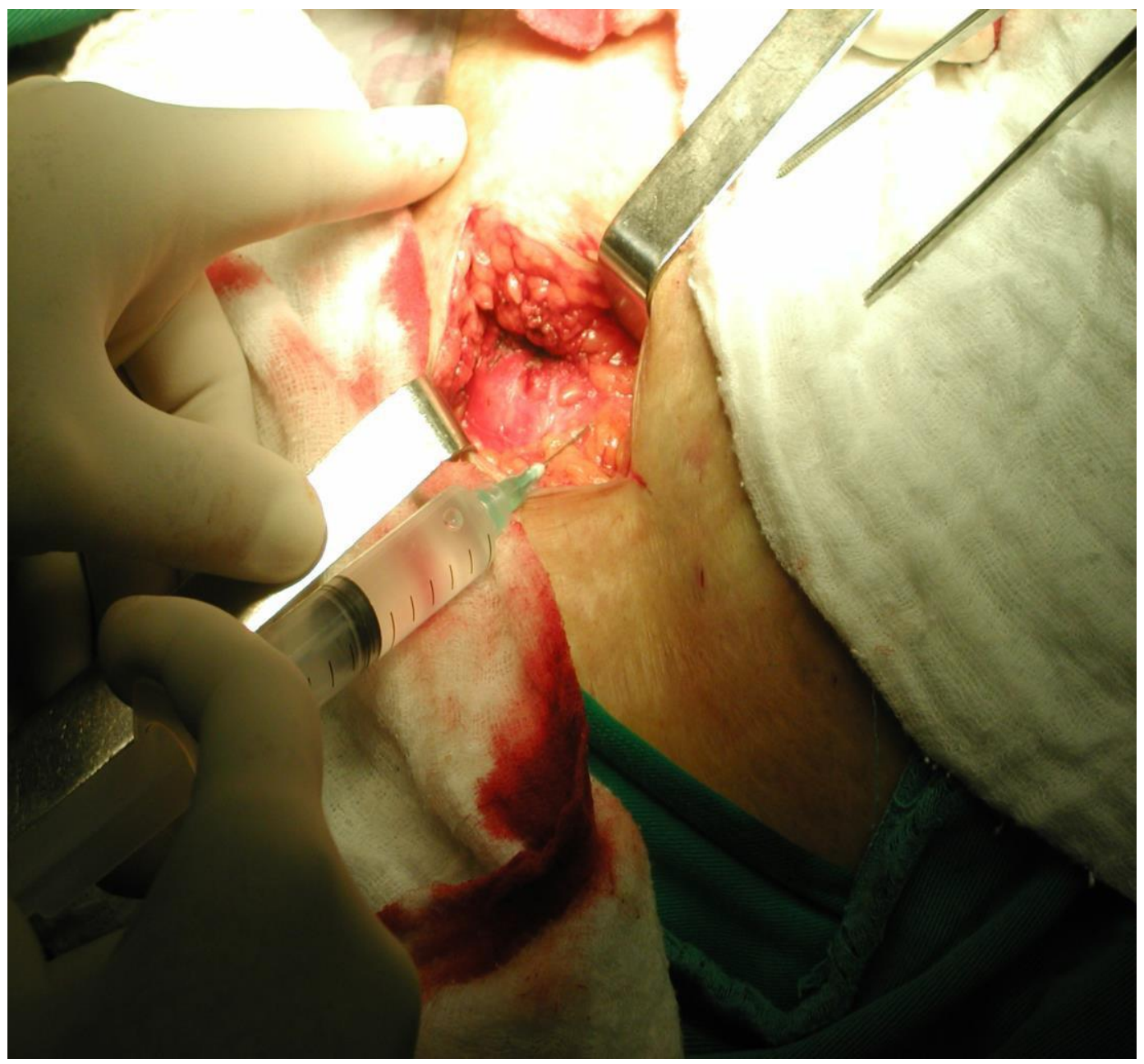

Figura $\mathbf{N}^{\circ}$ 2.- Infiltración del anestésico a nivel del cuello del saco herniario. 
Las técnicas quirúrgicas que deben utilizarse para la región inguinal son: Mac Vay, Shouldice, Bassini modificado, que tienen como finalidad reparar la pared posterior o piso del conducto inguinal y de ser necesaria, la utilización de prótesis, con la técnica de Lichtenstein.(Ver Fig. 3 y 4).

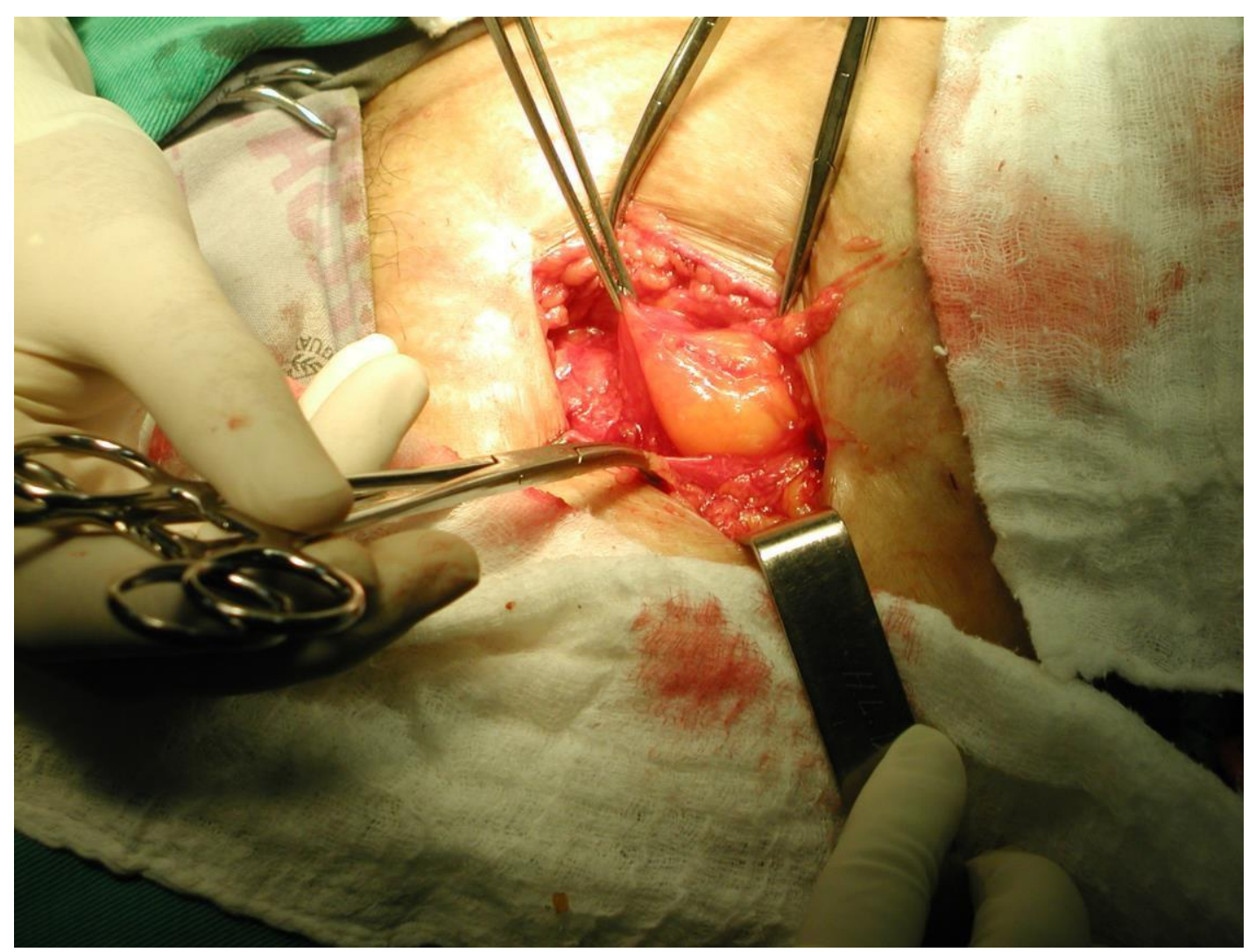

Figura $N^{\circ}$ 3.- Identificación y liberación del saco herniario de los elementos Inguinales. 


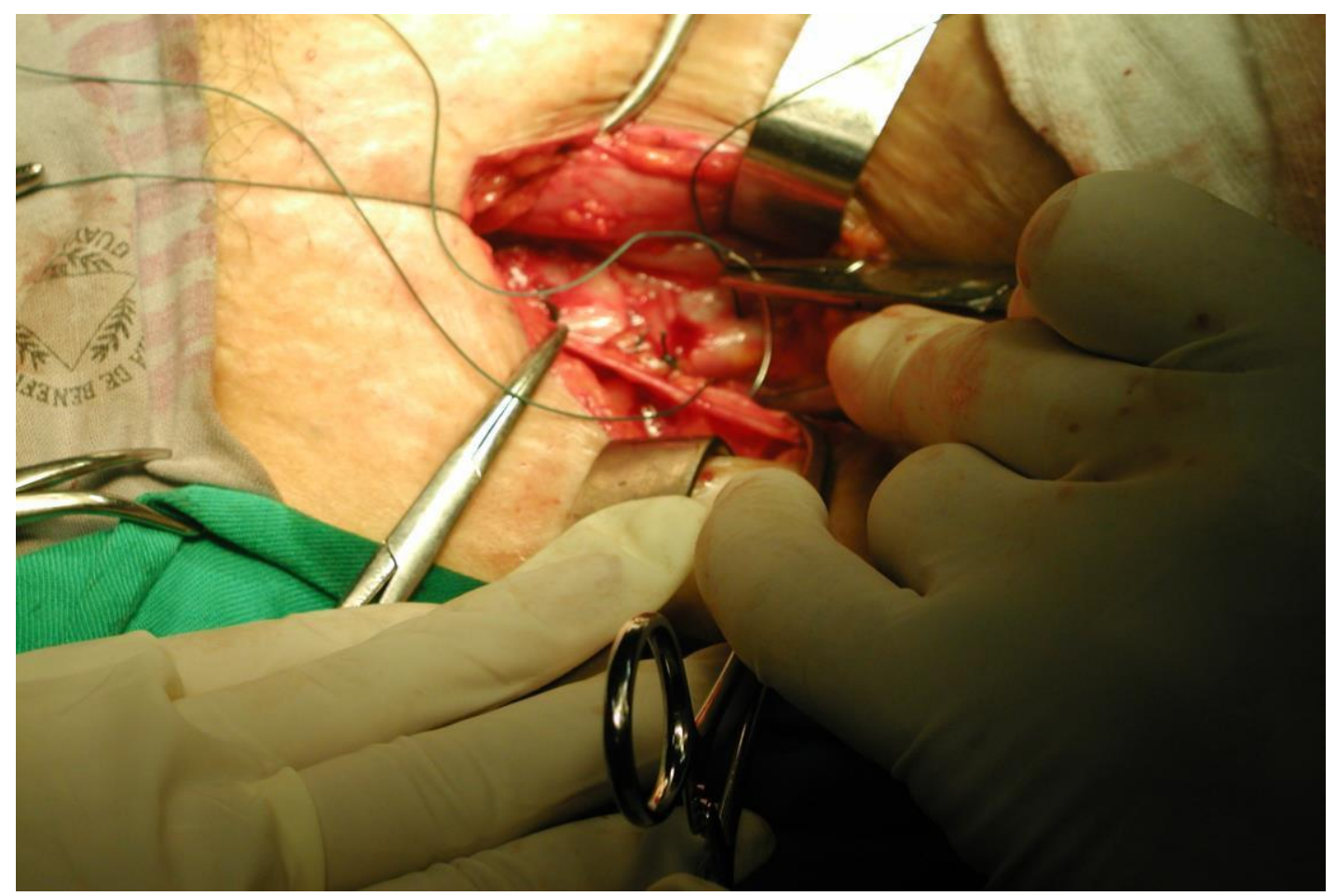

Figura $N^{\circ}$ 4.- Reparación del piso del conducto inguinal por la técnica de Bassini modificada.

La técnica anestésica para otras hernias de la pared abdominal consiste en:

1. Infiltración del anestésico, cubriendo área del procedimiento

2. Infiltrar a nivel del saco herniario y en alguna otra área álgica y que consecuentemente requiera anestésico

3. Siempre debe efectuarse aspiración frecuente para evitar inyectar anestésico directamente en un vaso sanguíneo.(Ver Fig. 5) 


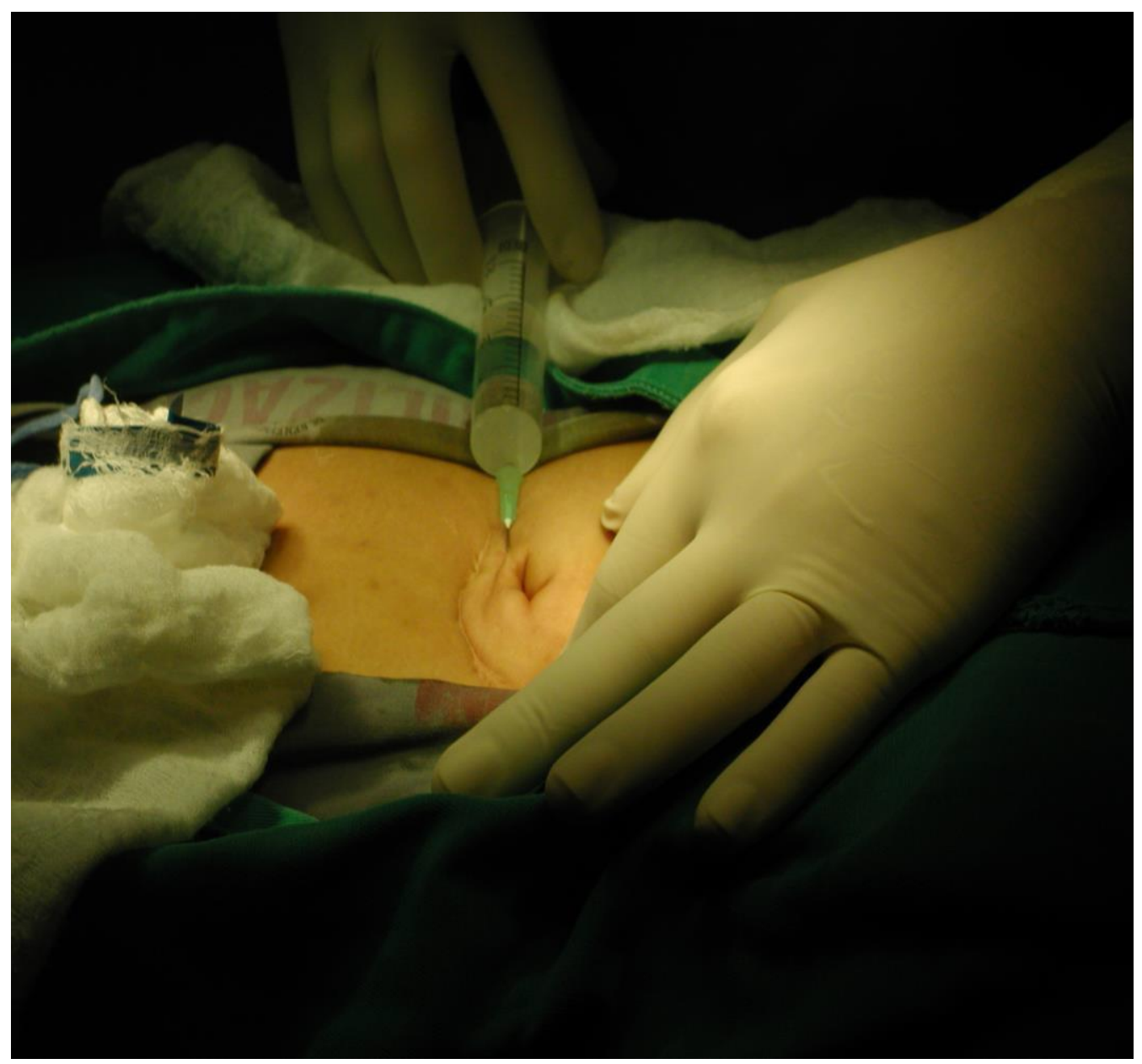

Figura $N^{\circ}$ 5.- Infiltración periumbilical del anestésico para reparar una Hernia Umbilical

\section{Recidivada}

Las técnicas quirúrgicas que se utilizan para otras hernias de la pared abdominal son:

STONE, CHALECO DE MAYO Y LICHTENSTEIN. (Ver Fig. 6, 7 y 8). 


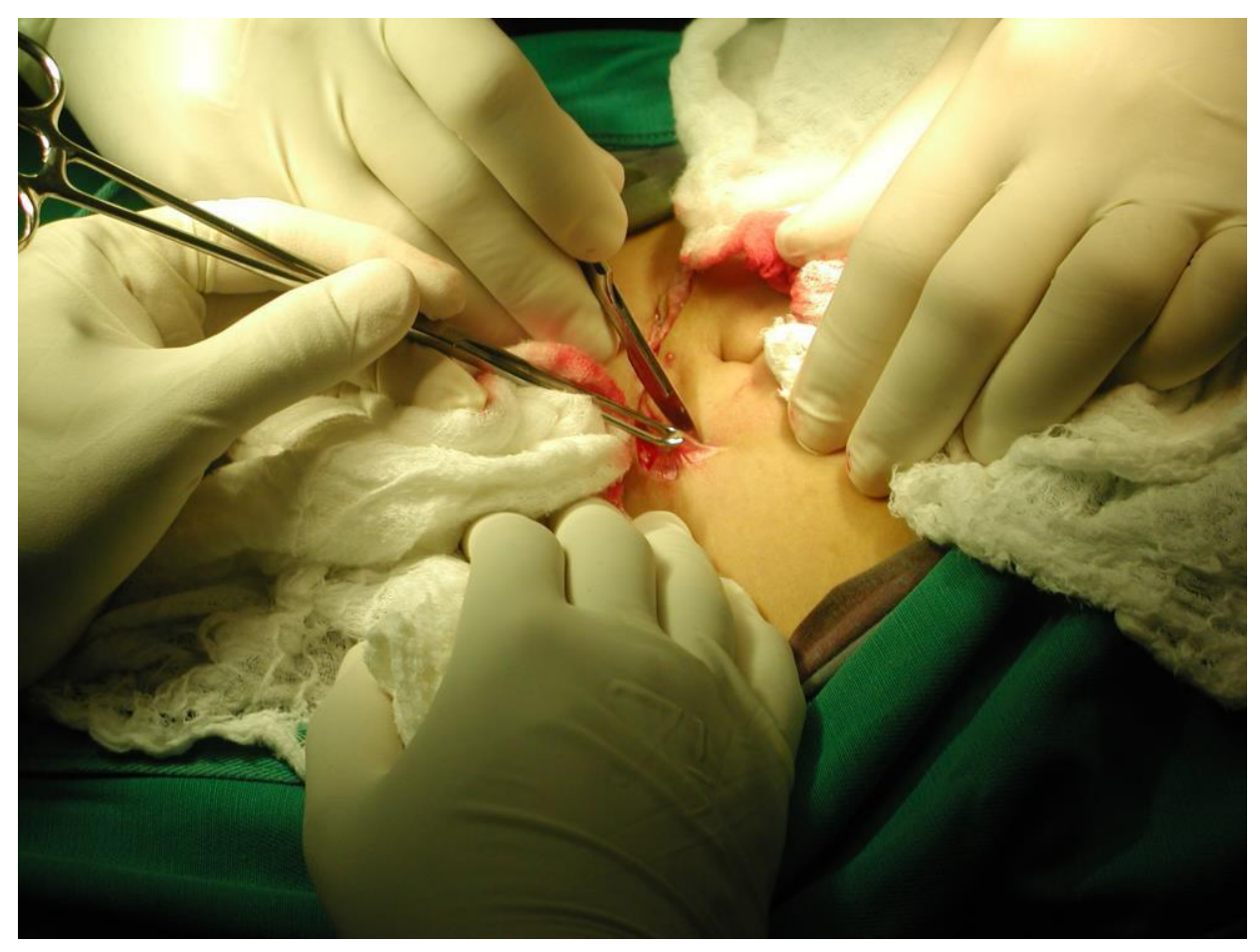

Figura $N^{\circ}$ 6.- Exéresis de cicatrizanterior.

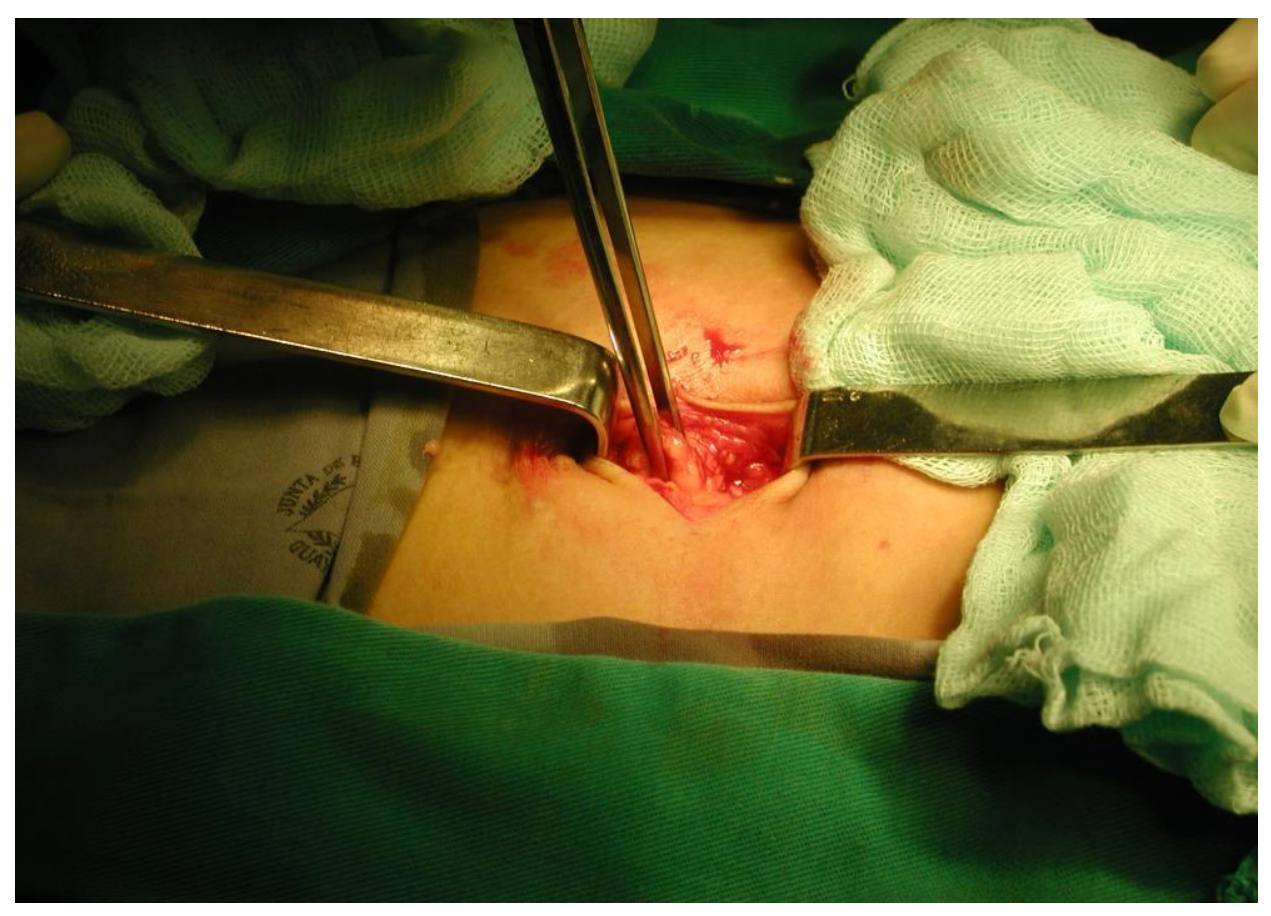

Figura $N^{\circ}$ 7.- Presencia del saco herniario 


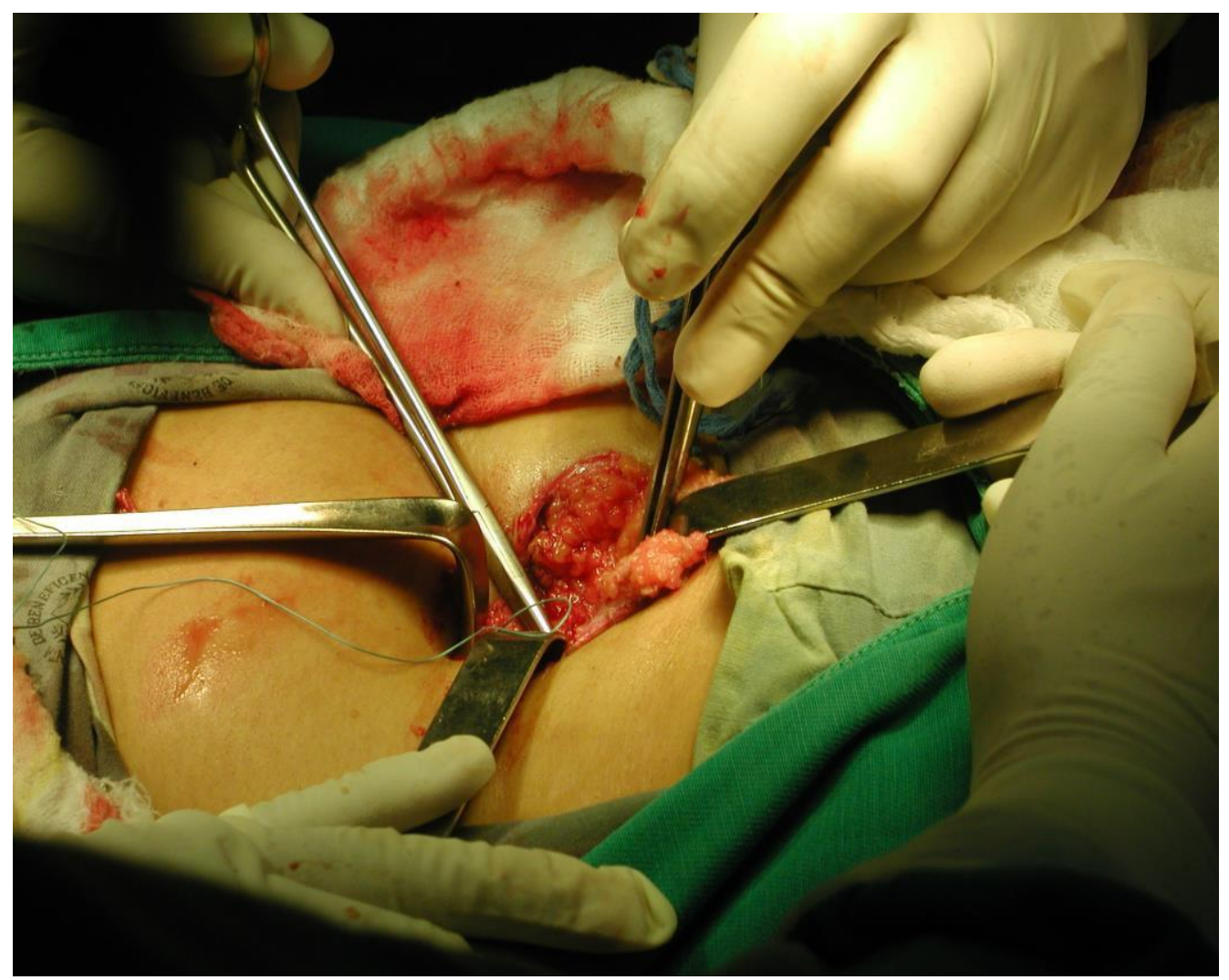

\section{Figura $N^{\circ}$ 8.- Reparación del defecto herniario mediante técnica de Stone}

\section{Clasificación de agentes anestésicos locales}

Los agentes anestésicos locales son: ésteres y amidas.

Los ésteres son inestables en solución, se hidrolizan rápidamente por la colinesterasa del plasma están asociados a fenómenos alérgicos y reacciones de hipersensibilidad. En este grupo se incluyen: cocaína, procaína, ametocaina y cloroprocaina.

Las amidas en cambio, son estables en solución y se metabolizan lentamente por las amidasas hepáticas, las reacciones de hipersensibilidad son sumamente raras. En este grupo se incluyen: lidocaina, prilocaina, mepivacaina y bupivacaina. 
El mecanismo de acción de los anestésicos locales, es produciendo interrupción reversible de la conducción de impulsos en los nervios periféricos, además, son inactivos en tejidos con pH ácido, por reducción en la liberación de bases libres, tal como ocurre en los abscesos.

Para la selección del anestésico local adecuado, debe considerarse que la lidocaina es la mejor opción para este propósito, porque se encuentra más disponible en nuestro medio, actúa más rápido y tiene duración de su efecto de 70 a 140 minutos.

Comúnmente encontramos lidocaina simple al $2 \%$ y lidocaina al $2 \%$ con epinefrina 1 en 200,000, las mismas que al diluirlas en un volumen similar, de solución salina isotónica o lactato de Ringer las convertimos en soluciones al 1\%. La dosis máxima permisible es de 7mg.Kg. para la lidocaina con epinefrina y de 3mg.x Kg. para la lidocaina simple.

La lidocaína es relativamente libre de efectos tóxicos y colaterales a dosis e infiltración adecuada. Las reacciones sistémicas se pueden en presentar probablemente por no seguir los correctos lineamientos. Los signos y síntomas son: adormecimiento perioral, gusto metálico, mareo, fotopsias, mioclonías, pérdida de conciencia, convulsiones, coma, paro respiratorio y paro cardiocirculatorio. El tratamiento de una intoxicación por anestésicos locales es básicamente de soporte mientras se espera que disminuyan los niveles plasmáticos. Consisten en: vía aérea permeable, ventilación con $\mathrm{O} 2$ al 100\%, soporte circulatorio con coloides y cristaloides, anticonvulsivantes como benzodiacepínicos o barbitúricos, simpático miméticos y de ser necesario cardioversión.

La aplicación de esta técnica anestésica es conveniente por las ventajas señaladas para el paciente y cabe recalcar también su beneficio económico, en lo que ahora se utiliza mundialmente como cirugía ambulatoria u hospitales del día, lo que consecuentemente representa una disminución manifiesta en el rubro correspondiente a estancia hospitalaria. 


\section{Conclusiones.}

Las hernias de la pared abdominal pueden ser intervenidas quirúrgicamente con la ayuda de la anestesia local. Los beneficios son múltiples, tales como: cirugía ambulatoria, menor tiempo de estancia hospitalaria, menores costos, disminución de ansiedades del paciente. La aplicación del procedimiento debe realizarse con el consentimiento informado y debidamente explicado, para que el paciente conozca que no va a sentir dolor, pero podrá sentir movimientos que realizará el cirujano para corregir el defecto herniario. Es muy útil en pacientes envejecientes, que tienen alguna comorbilidad que impida recibir otro tipo de anestesia, que requiera movilización inmediata o que deba tener ingesta de alimentos sin interrupción.

\section{Bibliografía.}

1. Aledjan JJ, Bruellep et de la Coussaye JE; "Pharmacologie des anesthèsiques locaux". Encycl. Mèd. Chir (Elsevier, paris-france) Anesthèsie Reanimation, 36-320-a-10, 1996, 16 p.

2. Alvarado Bestene J, et al.: “Manual de Cirugía”, la ed. Santa Fé de Bogotá: ceja, 199. Cap. II p. 17994.

3. Amid. Pk; Shulman, Ag; Lichtensteinil;"Local anesthesia for inguinal hernia repair-step-by procedure" Ann. Surg., 1994, 220, 6: 735- 737.

4. Bendavid r: "The Shouldice repair. Inguinal hernia repair, eds: Schumpelickv, Wantz ge Basel, Karger, 1995 , pp $122-134$.

5. Escallón Mainwaring J; "Herniorrafia inguinal. Técnica de Shouldice modificada”. Rev. Colomb. Cir. 1987; 2: $69-74$.

6. García Gil, JM: “Cirugía de las hernias en el anciano”. Cir. Esp. 1995, 57, 2: 98 - 101.

7. García Pulido R, Casanova Pérez PA, Fournier Villa HI. "Herniorrafia inguinal libre de tensión con la técnica de Lichtenstein. Cir. Ge 2002; 24(3): 209 - 12. 
Rafael D. Espinosa-Cucalon; Clara O. Jaime-Game; Antonio Romero-Vargas; Carmen L. Marín-Soria

8. Gianetta E, Cuneo S, Vitale B, Carmen G, Marini P, Stella M. “Anterior tensión - free repair of recurrent inguinal hernia under local anestesia: Ann Surg. 2000. Jan, 231(1); 132 - 134.

9. González Lozano, R; “Clínica de cirugía y gastroenterología”. Monterrey, n.l.c.p. 64710. México 1998.

10. Lezcano H, Delgado JR. “Farmacología de los anestésicos locales”. Rev. Arg. Anest. 1995; 53, supl.:27 $-33$.

11. Lichtenstein IL, “Hernia repair without disability”. St. Louis: CV Mosby, 1970-

12. Marin Morales J; Gallardo García A; Pérez Ramírez C, Martínez Diestre M: 'Tratamiento quirúrgico ambulatorio de la hernia inguinal bajo anestesia local más sedación”. Cir. Esp. 1994, 55, 1: 33 - 36.

13. Martínez C, Núñez JR, Jorgensen TW, Sanz R, Ruiz M, Pastor L. "Herniorrafia de Bassini en la hernia inguinal primaria”. Cir. Esp. 1996; 59(6): 509 - 513.

14. Milligan KR, Convery PN, Weir P, Quinn P, Connolly D. “Anesthesia and analgesia”. Aug 2000; 91 (2) p $393-7$.

15. Obiols Aldoma J.A; Guillaumes Furnes, S.; Trias Rubies R.:"La anestesia local permite reducir la morbilidad y la estancia hospitalaria en la cirugía de la hernia inguinal". Cir. Esp., 1994, 56, 4: 307 311.

16. Talenian DL, "Los anestésicos locales. Beneficios, riesgos y uso. Curso de actualización en anestesiología. (ASA). Ed. Lippincott - Raven. 1997; Vol 25, cap 17.

17. Welsh D, Alexander M: “The Shouldice repair" Surgical Clinics of North América, Vol 73, no 3, June 1993, pp $451-469$. 